\title{
A Computed Tomography (CT) wish list, from a medical physicist's perspective
}

\author{
Zoe Brady $\cdot$ Paul Einsiedel
}

Published online: 21 March 2012

(c) Australasian College of Physical Scientists and Engineers in Medicine 2012
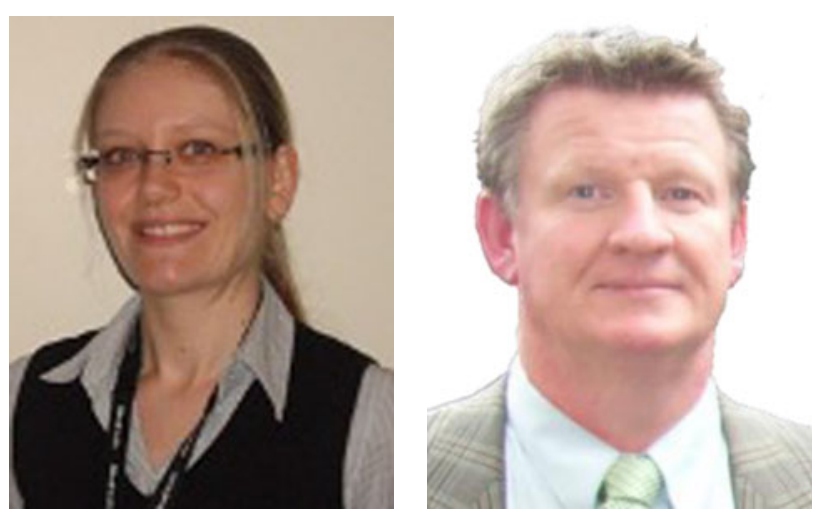

Significant advances in computed tomography (CT) technology have been made since the first clinical images were published in 1973. The initial clinical applications were brain scans where the patient's head was immobilised by a water bag that compensated for the large changes in flux adjacent to the head. Body imaging became feasible as improvements led to better image quality and reduced scan times. Improved computing technology has allowed for image reconstruction, including rendering and 3D reconstruction, to all occur in real time. Perfusion studies, cardiac imaging and CT angiography are now common and

\section{Z. Brady $(\square)$}

Radiology Department, The Alfred, P.O. Box 315,

Prahran, VIC 3181, Australia

e-mail: z.brady@alfred.org.au

\section{Z. Brady}

Applied Physics, RMIT University, GPO Box 2476V,

Melbourne, VIC 3001, Australia

\section{P. Einsiedel}

Department of Radiology, The Royal Melbourne Hospital, Grattan Street, Parkville, VIC 3050, Australia interventional procedures can be performed using CT fluoroscopy guidance. Some of the major scanner developments that have contributed to this progress are slip-ring technology, which facilitated helical scanning, and multidetector CT systems with capabilities for volume scanning. In addition, increasingly complex X-ray tube designs and novel CT detector materials and configurations have also contributed to changes in CT imaging. Dose reporting began to address concerns raised regarding the risks from radiation exposure and other practical dose reduction measures such as the adaptation of parameters to the patient size were implemented. This included modulating the tube current throughout the scan. More recent CT developments include dual-energy imaging and iterative reconstruction techniques, which are used instead of or in combination with traditional filtered back-projection image formation. All of these improvements in technologies combined have resulted in dose reductions for some CT procedures in the order of $90 \%$ !

The changes to image quality, dose and the way in which CT scans are performed have been extensive. However, there are always further enhancements that can be made, especially as user requirements or objectives change. For diagnostic medical physicists, there will always be a wish list of what we would like on a CT scanner. This is largely driven by the work that we undertake and the needs of our colleagues, who request, perform or interpret CT scans. Inevitably, achieving the best health outcome for the patient is the primary concern. The wish list is also influenced by current opinions and attitudes towards CT imaging. Hence, the desirable features for a CT scanner are discussed below, with the rationale for these first explored. A small straw poll of radiologists and radiographers from a variety of workplaces was also undertaken to gauge opinions. 
Current articles regarding the use of CT typically start with a statement on the population dose attributable to CT scanning and the rate at which it is increasing and/or the significant growth in the number of CT scans undertaken annually. This is for good reason. In most hospitals or diagnostic imaging practices, there is a growing perception that CT imaging may be over-utilised and hence some proportion of the radiation exposure is unwarranted. It is difficult to quantify the number of unjustified CT scans undertaken. When the question was posed in the straw poll, radiologists felt that the rate of unnecessary CT imaging depended on the clinical setting (e.g. emergency department or whether a Medicare rebate was available) and that in fact paediatric imaging in dedicated children's hospitals may all be appropriately justified. However, some radiologists still believed that the percentage of CT scans that are unnecessary ranged from 20 to $70 \%$. Radiographers felt the situation was not necessarily as bad and that unjustified CT scans accounted for $0-20 \%$ of all CT imaging. The figure is unlikely to be as low as $0 \%$ or as high as $70 \%$, but it is clear that this issue is significant. Analysis of Medicare records confirms that Australian CT imaging rates are increasing, with an annual growth rate of $8.5 \%$ [1]. Additionally, a recent international survey found that CT accounts for almost half of the collective population dose from diagnostic procedures $(43 \%)$ while constituting less than $10 \%$ of the number of procedures performed [2]. Therefore, CT is a high dose diagnostic modality, which is being increasingly utilised. Of note, a survey [3] conducted in 1997 by the Australian Radiation Laboratory (now the Australian Radiation Protection and Nuclear Safety Agency, ARPANSA) found that Australia was "well endowed with CT scanners" and ranked only behind Japan in the number of CT scanners per million people.

The increase in the number of $\mathrm{CT}$ examinations being performed could also be attributed to the cost and availability of CT hardware. The level of R\&D that has been undertaken over the past 20 or so years and the increased competition amongst the major vendors has seen the cost of CT equipment decrease relatively, making it more affordable and accessible. As a consequence, it is now not uncommon to see multiple CT scanners being placed in hospitals and major radiology clinics which obviously improves the availability of the imaging modality and increases the number of scans being performed.

With the uptake in CT imaging and the increasing awareness of potential stochastic risks from radiation exposure, medical physicists are being asked more frequently about CT doses and the associated or potential risks by radiographers, radiation medical practitioners, referrers and members of the public. In the straw poll, the majority of radiologists and radiographers also felt there was greater awareness amongst the general public about radiation. Some of this perhaps stems from the recent Fukushima nuclear reactor incident in Japan in 2011 or possibly from increased media reporting or access to the internet by the general public dealing with $\mathrm{CT}$ and radiation doses. A new professional group may have possibly been created, namely, the "Google Medical Physicist". However, we would hope that overall patients are generally better informed. In some cases, patients demand that their CT scan be performed at or under a specific dose. Anyone working in a radiology department knows that a submillisievert dose for a chest CT scan on a large adult patient is not yet feasible. However, such low dose CT imaging is promoted by some manufacturers. One of the radiologists queried in the straw poll drew a correlation between manufacturer advertising, often cardiac related, and that general practitioners (GPs) only paid attention to cardiac CT doses. Given the recent Report to the Professions [4] which highlighted unnecessary CT referrals by GPs, awareness of the radiation dose and hence the ability to make an informed decision regarding the justification of the scan should be applicable to all CT procedures and not just those that are popular and well advertised.

In recent years, a greater focus has been on paediatric CT imaging because of the higher risk to children from radiation exposure. However, there is evidence that the radiation exposure risks in middle age are underestimated in the standard initiation-based model and that when cancer promotion processes are taken into account the risks may be up to twice as high [5]. This reinforces efforts to lower doses for children and people in the middle age groups. The straw poll indicated that justification of CT imaging may be more conservatively and hence better performed in children's hospitals, but this emerging data on middle age risk demonstrates that practices that scan adults also need to become more diligent in enforcing justified imaging. This could occur from improved education of the referring practitioners on the radiation doses, risk and need to justify the use of CT for imaging and diagnosis. In most cases this may require better access and education as to what resource a medical physicist can provide to medical professional groups that may refer for imaging services.

Within the current climate of rising and perhaps unwarranted use of CT imaging, the radiation protection principles of justification and optimisation are especially important. To appropriately justify the scan, the referrer requires knowledge of both the risks from radiation exposure, which requires the dose to be known, and the potential benefits for the patient's management. To reduce the risks, the dose needs to be minimised, which will affect the image quality. Therefore, CT technology must provide avenues for addressing each of these issues. Dose optimisation tools should positively influence image quality and the dose from a scan must be reported and readily retrievable at a later date. Manufacturers are acutely 
aware of these issues and are constantly offering solutions. However, in reality what we currently have clinically is still far behind what is commercially available. Dual energy CT scanners are installed in hospitals in Australia, but some without the costly added features to fully utilise use of the second X-ray tube, for example dual energy scanning. Iterative reconstruction which can both lower dose and improve image quality is available, but cannot be retrofitted to some older scanners. Structured dose reporting (SDR), which allows more complete dose records is available on some platforms, but not yet integrated with picture archiving and communication systems (PACS). Therefore, currently medical physicists are challenged to find a workable solution based on the age and limited equipment software to record, monitor and document patient doses from CT scanners. Dedicated solutions that are able to automatically track and store CT scanner parameters and patient dose appear to be only available at equipment replacement provided the specific requirements to include such functionality have been addressed in the tendered solution. Given the intent to facilitate justification and ensure optimisation of dose and image quality, here are some of things that would improve CT scanners that are in current use.

\section{Dosimetry information}

On most, if not all, clinical CT scanners the volumetric CT dose index $\left(\mathrm{CTDI}_{\mathrm{vol}}\right)$ and dose length product (DLP) are shown as projected values following the CT localiser radiograph before the scan is performed. Individual values resulting from each component of the examination and total values are also displayed in a dose report screen at the conclusion of the scan based on the parameters used. On many scanners this information has to be captured using a screen shot to then send through to PACS as a record for the patient. Not all practices capture the dose report and therefore this information is retained for only as long as the patient's scan is stored on the CT workstation (a few days to weeks depending on the throughput of the scanner). This makes it difficult for retrospective dose assessment based on the dose indicators. Furthermore, when the dose report screen is sent to PACS, it can not be easily interrogated for audit purposes, in particular accessing data for deriving local diagnostic reference levels (DRLs). Structured dose reporting will address some of these issues, but it is yet to be widely implemented and where it is used, PACS compatibility issues still occur. It is also possible to use optical character recognition to retrieve information from the screen-captured dose reports. However, these types of workarounds should not be necessary. Ideally, the solution to overcome these difficulties is to have the SDR supported by all PACS vendors and stored along with all other patient images and reports, with an added PACS functionality to allow it to be filtered and mass exported for analysis. Alternatively, the SDR could be directly handled by the CT modality and stored at the modality or remotely for future analysis. This latter option would be the least preferred as minimal dosimetry information would then be retained with the patient on PACS for future reference.

In terms of the analysis process, the delivered dose depends on, and is not limited to, the scan length and patient size. It is not uncommon for the CTDI $\mathrm{v}_{\mathrm{vol}}$ and DLP to double for the same procedure performed on two different patients. While scan length can be reported, the patient weight is not. Consequently to assist in analysis of patient dose records it would be ideal to report both scan length and patient weight for each CT scanning protocol performed on a patient. This may require manual entry of the patient weight into the $\mathrm{CT}$ modality, although the preferred option is to have vendors include scales in their tables with the measured weight automatically populated in the SDR. This would also have the added advantage of warning the radiography staff when table weight limits have been exceeded.

A limitation of the CTDI ${ }_{\mathrm{vol}}$ is for scans performed with tube current modulation where only a single CTDI $_{\mathrm{vol}}$ value is calculated. It is often unclear which parameters this value is based on, since the tube current changes throughout the scan and may not be readily retrievable. To allow for an improved assessment of dose, in addition to reporting the average $\mathrm{CTDI}_{\mathrm{vol}}$, consideration could also be given to reporting local $\mathrm{CTDI}_{\mathrm{vol}}$ in anatomical regions for "high radiation risk organs". This would require some further smart technology to be introduced to the scanner to automatically locate these regions from the scout view and report the corresponding CTDI $_{\mathrm{vol}}$. This approach could be particularly useful in light of the current $\mathrm{CT}$ technologies where vendors are varying pitches on the fly throughout an exam or modulating and even switching off the X-ray beam over high risk organs. Further, variants such as the size-specific dose estimate (SSDE) recommended by the American Association of Medical Physicists (AAPM) [6] or even size-specific organ absorbed dose estimates based on the CTDI ${ }_{\mathrm{vol}}$ [7] may be useful additions to the dose information displayed on the scanner. An automatically calculated lateral and anteriorposterior dimension (recognising skin edge) for each patient would also assist in size-specific dosimetry.

Consideration could also be given to supplying an effective dose estimate for an examination, although it is not recommended as an appropriate quantity for the retrospective assessment of individual exposures [8]. An alternative option, if organ absorbed dose estimates for individual patients are available, is the quantity "effective risk", which uses age-, gender- and tissue-specific lifetime cancer risks to calculate a weighted sum of tissue and organ equivalent 
doses [9]. This is a more intuitive and easily interpretable quantity, as it expresses the dose in terms of the risk of radiation-induced cancer based on current knowledge and therefore may contribute towards improved justification of scans through simplification of risk/dose expression.

For helical CT scans, there is an additional length required at each end of the scan to allow for data interpolation. This "over-ranging" length is currently not evident in either the localiser tomogram or scan images. In addition, on current scanners it is not clear if the reported $\mathrm{CTDI}_{\mathrm{vol}}$ or DLP includes the over-ranging. It would be extremely useful to have an indication of the "imaged" length, as well as the full length, including over-ranging, to which the patient has been exposed. This would be particularly important in imaging of pregnant patients, where more precise collimation is required to minimise/optimise foetal doses.

\section{Optimisation tools}

National DRLs will soon be established by ARPANSA for CT examinations. These reference levels will be beneficial for narrowing the dose distribution across Australian CT practice and will provide instrumental guidance at the local practice level. With the availability of DRLs, it would be useful to have these entered in the CT workstation software, so that the projected values of $\mathrm{CTDI}_{\mathrm{vol}}$ and DLP can be automatically assessed. An alert or notification could be provided to the user if the projected values are high in comparison with the reference level. This would raise user awareness of doses as the operator of the CT scanner would need to justify why the potential dose from the scan(s) significantly exceeds the accepted DRLs. This may reduce the occurrence of errors specifically related to the selection of the exposure parameters, especially for procedures involving CT perfusion or CT fluoroscopy where deterministic effects can be experienced. Some manufacturers are in the process of making these tools available and it would be worthwhile to make them mandatory on all scanners. Furthermore, allowing auditing and analysis of temporal trends by extracting dose data would also be a convenient feature.

Information on the dose is essential, but image quality feedback is equally important. Building in an automatic assessment of image quality would be an interesting and beneficial tool to have. However, the complexity of this may prevent it from being realised clinically. Alternatively, an optional tool could be made available to allow the user to score the image quality using a simple scale. Further, for some vendors, image quality assessment is performed either at installation or at routine intervals, however, is only available via the vendor's unique service tool/log-on.
Consideration should also be given to allow the medical physicist access to this image quality module. This would allow quicker, more frequent and consistent spot checks on image quality to be undertaken. Noting, for busy radiology departments, access to the CT scanner for routine medical physics testing can be rather problematic. The outcome is that these records could be extracted over time and some correlation may be drawn between patient size and/or protocol parameters to assist in the continual process of image quality improvement. Ideally, reference levels would be available for both dose and image quality.

Reviewing and changing protocol parameters to lower dose and improve image quality is also fundamental to the optimisation process. Therefore, it is necessary to have the ability to easily extract, in a user-friendly format, the protocols programmed on a scanner. Downloading of protocols by the end user is currently not accessible on all scanners. There are tens of parameters that can be defined per protocol and typically more than 100 protocols per scanner making the task of protocol evaluation onerous. Without being able to easily compare protocols, it is a conceivable situation that a department with multiple scanners of the same model may have different protocols for an examination when the intention is for them to be the same. From a quality control perspective, it is crucial that access to protocols is made easy and even simplifies the comparison of parameters.

\section{Terminology standardisation}

A significant hindrance to understanding CT dose and the parameters that impact on dose is the lack of consistent CT terminology. For example, the two dimensional localiser radiograph performed at the start of a CT scan is referred to as the scout, surview, topogram, tomogram or scanogram depending on which brand of CT scanner is used. More importantly, all modern scanners are equipped with tube current modulation and the reference parameter differs significantly between manufacturers from being a reference image, to a tube current value to an indicator of the noise in the image. Since this reference parameter directly affects image quality and patient dose on almost every examination, the disparity between manufacturers can be problematic in truly achieving optimisation.

Expertise with a range of vendors and also different models from the same vendor is necessary to have even the most basic understanding of current CT technology. The complexity of this issue is evidenced by the CT lexicon issued by the AAPM [10], recognising the need to standardise terminology. An unnecessary barrier exists when there is the need to translate CT terms so that they can be more readily understood. From the straw poll, CT supervisor radiographers generally indicated that they would be 
comfortable working across different CT platforms. However, they felt that this was a significantly harder task for rotating radiographers who were not dedicated to $\mathrm{CT}$ and that ongoing education for multi-vendor departments would be challenging.

\section{Conclusions}

This is by no means an extensive list of what could be included on a CT scanner. Some desirable features have been discussed in the context of why they are needed and manufacturers are regularly delivering new solutions to the identified issues. However, scanner features which lead to improved image quality and lower dose should not only be available on "high end models" but should be part of any base model, and not even offered as an "extra cost option". It is important that the clinical implementation of beneficial tools more closely aligns with commercial availability. Both radiologists and radiographers questioned in the straw poll agreed that faster scanners in terms of processing, lower doses, improved image quality and iterative reconstruction were the priority areas to be addressed. Ultimately, patients, who are our primary concern, will benefit if these types of features are implemented on new and old model scanners. To ensure these features are delivered with any new purchase it is imperative that medical physicists have input into the decision making process at the time of purchase. By not doing so, you may be left trying to invent timely and costly workarounds to achieve optimal medical physics support and possibly legislative compliance.

\section{References}

1. Brady Z, Cain TM, Johnston PN (2011) Paediatric CT imaging trends in Australia. J Med Imaging Radiat Oncol 55:132-142

2. United Nations Scientific Committee on the Effects of Atomic Radiation (UNSCEAR) (2010) Sources and effects of ionizing radiation, Vol I. UNSCEAR 2008 Report to the General Assembly. Annex A: Medical Radiation Exposures. United Nations, New York

3. Thomson JEM, Tingey DRC (1997) Radiation doses from computed tomography in Australia. Report ARL/TR123. Commonwealth Department of Health and Family Services, Australian Radiation Laboratory, Canberra

4. Professional services review (PSR) (2010) Report to the Professions 2008-2009. Canberra

5. Shuryak I, Sachs RK, Brenner DJ (2010) Cancer risks after radiation exposure in middle age. J Natl Cancer Inst 102(21): $1628-1636$

6. American Association of Physicists in Medicine (AAPM) (2011) Size-specific dose estimates (SSDE) in paediatric and adult body CT examinations. AAPM Report No. 204. College Park

7. Turner AC, Zhang D, Khatonabadi M, Zankl M, DeMarco JJ, Cagnon CH, Cody DD, Stevens DM, McCollough CH, McNittGray MF (2011) The feasibility of patient size-corrected, scanner-independent organ dose estimates for abdominal CT exams. Med Phys 38(2):820-829

8. International Commission on Radiological Protection (ICRP) (2007) The 2007 Recommendations of the international commission on radiological protection. ICRP Publication 103. Ann ICRP 37(2-4):1-332

9. Brenner DJ (2008) Effective dose: a flawed concept that could and should be replaced. Br J Radiol 81(967):521-523

10. American Association of Physicists in Medicine (AAPM) (2011) AAPM CT Lexicon Version 1.1. AAPM, College Park 